\title{
Design and Development of the Efficient Anguilliform Swimming Robot - MAR
}

\author{
Konstantin Struebig, Behzad Bayat, Peter Eckert, Anouk Looijestijn, Tim C. Lueth, and Auke J. Ijspeert.
}

\begin{abstract}
Propulsion of swimming robots at the surface and underwater is largely dominated by rotary propellers due to high thrust, but at the cost of low efficiency. Due to their inherently high speed turning motion, sharp propeller blades and generated noise, they also present a disturbance to maritime ecosystems. Our work presents a bio-inspired approach to efficient and ecofriendly swimming with moderate to high thrust. This paper describes the concept, development and experimental validation of the novel anguilliform robot MAR. With 15 elements making up the $0.5 \mathrm{~m}$ long propulsive section and driven by a single, speed-controlled brushless DC motor (BLDC), the robot creates a smooth continuous traveling wave for propulsion. Steering and autonomy are realized by an actuated head with integrated batteries that serves as a front-rudder. Almost neutral buoyancy paired with individually actuated pectoral fins furthermore enable submerged swimming and diving maneuvers. MAR accomplished high thrusts at a moderate power consumption in first performance tests. The achieved maximum velocity and the speed related efficiency (defined as the achieved speed over the power consumption $\mathrm{m} / \mathrm{Ws}$ ) did not fulfill the expectations in the first tests (in comparison to commercial rotary thrusters), which can be largely attributed to the spatial limitations and an imperfect test setup. Nevertheless, the potential towards highly efficient and high thrust propulsion is visible and will be further investigated in future efforts.
\end{abstract}

Index Terms-Anguilliform, Swimming, Helix, Traveling Wave, Fish-like, Bioinspired, Robot

\section{INTRODUCTION}

A QUATIC propulsion is currently predominantly realized through the use of screw propellers. Developed in the 19th century, these propulsors have been radically optimized over the years and represent the widely adopted standard. Due to performance limiting effects like cavitation, eddy formation and ventilation however, the propeller efficiency lies between only $50 \%$ and $75 \%$ [1]. Another problem is the disturbance of maritime ecosystems through impacts with animals and aquatic flora as well as noise. Collisions with whales resulting in severe injuries, destruction of seagrass that serves as a habitat for smaller organisms and noise radiation influencing fish behaviour are only a few of the measurable negative consequences. A more natural approach is inspired by fish, that use undulation and oscillation rather than rotation to generate propulsion with efficiencies of up to $90 \%$ [2]. If such fishlike motion could successfully be transfered onto a mechanical

Konstantin Struebig and Tim C. Lueth are with the Institute of Micro Technology and Medical Device Technology at the Technical University of Munich. Contact: Konstantin.Struebig@TUM.de.

Behzad Bayat, Peter Eckert and Auke J. Ijspeert are with the Biorobotics Laboratory at École Polytechnique Fédérale de Lausanne.

Anouk Looijestijn is with the Department of BioMechanical Engineering at Delft University of Technology. system, the efficiency of marine robotics and transport could be significantly increased, while lowering the negative impact on the environment.

\section{A. Fish Locomotion}

The movement of fish can be categorized into two main categories, namely body and caudal fin locomotion (BCF) and median/paired fin locomotion (MPF). BCF locomotion uses the movement of the body and the large caudal fin to generate strong propulsion resulting in high acceleration and top speeds. MPF locomotion relies on the undulation and oscillation of pectoral, dorsal and anal fins for better maneuverability [3]. Since the goal of this research is to generate strong and efficient propulsion, BCF locomotion is further investigated. Within this category, five types of swimming are defined. Anguilliform, subcarangiform, carangiform, thunniform and ostraciiform swimming differ from another regarding the declining percentage of the body that is used for propulsion and the degree of undulatory motion, that gradually changes into an oscillatory motion (Fig. 1). While anguilliform swimmers, like eel and lamprey, undulate their whole bodies, obstraciiform swimmers, like boxfish, only oscillate their caudal fin with the rest of their bodies rigid. Among these five types, the highest efficiency has been observed in anguilliform swimming, which is characterized by a traveling wave of body undulation along the entire body. The European Eel (Anguilla anguilla) proves this extraordinary movement efficiency of this locomotion type during its migration to the Sargasso Sea, which involves continuous swimming over several months without feeding [4]. The hydrodynamic benefits over other types have also been observed in simulations, based on the energy loss through vortex wakes downstream [5].

\section{B. State of the art}

Many research groups have studied aquatic locomotion and its implementation in a fish-like robot, starting with the first robot fish from MIT in the 1980s [7]. While the idea of

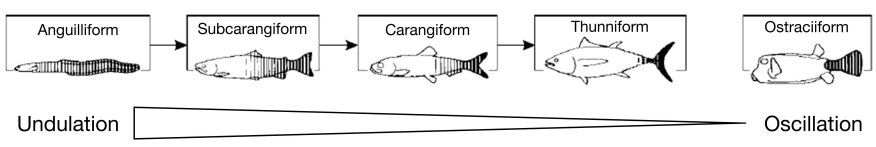

Fig. 1. The five categories of body and caudal fin (BCF) locomotion. The shaded areas indicate the part of the body that is actively generating thrust. With a decreasing fraction of the body length responsible for the propulsion, the undulatory movement transitions into an oscillatory movement. Adapted from [6]. 
TABLE I

AN OVERVIEW OF THE SPECIFICATIONS OF THE PRESENTED ANGUILLIFORM SWIMMING ROBOTS AND THE NEWLY DEVELOPED PROTOTYPE MAR. WHILE THE BATTERY SPECIFICATIONS OF Amphibious snake-like robot IS NOT AVAILABLE, THEY ARE MOST LIKELY SIMILAR TO THOSE OF Lampetra Artefact. OTHER ATTRIBUTES LIKE THE EFFICIENCY, RUNTIME AND THRUST GENERATION ARE QUALITATIVELY ESTIMATED.

\begin{tabular}{|c|c|c|c|c|}
\hline & $\begin{array}{c}\text { Lampetra } \\
\text { Artefact }\end{array}$ & $\begin{array}{c}\text { Amphibious } \\
\text { snake-like } \\
\text { robot }\end{array}$ & $\begin{array}{l}\text { Envirobot } \\
(6 \text { modules })\end{array}$ & MAR \\
\hline Length & $99 \mathrm{~cm}$ & $117 \mathrm{~cm}$ & $99 \mathrm{~cm}$ & $108 \mathrm{~cm}$ \\
\hline Weight & $1640 \mathrm{~g}$ & $6750 \mathrm{~g}$ & $3215 \mathrm{~g}$ & $7500 \mathrm{~g}$ \\
\hline Segments & 21 & 9 & 6 & 15 \\
\hline $\begin{array}{l}\text { Number of } \\
\text { actuators }\end{array}$ & 10 & 24 & 5 & 1 \\
\hline $\begin{array}{c}\text { DoF per } \\
\text { joint }\end{array}$ & 1 & 2 & 1 & 1 \\
\hline $\begin{array}{c}\text { Actuation } \\
\text { type }\end{array}$ & $\begin{array}{l}\text { Magnetic } \\
\text { actuator }\end{array}$ & $\begin{array}{c}\mathrm{DC} \\
\text { motor }\end{array}$ & $\begin{array}{c}\mathrm{DC} \\
\text { motor }\end{array}$ & $\begin{array}{c}\mathrm{DC} \\
\text { motor }\end{array}$ \\
\hline Control & CPG & $\begin{array}{c}\text { Serpenoid } \\
\text { curve }\end{array}$ & CPG & $\begin{array}{r}\text { Velocity } \\
\text { control }\end{array}$ \\
\hline Speed & $0.25 \mathrm{~m} / \mathrm{s}$ & $0.07 \mathrm{~m} / \mathrm{s}$ & $1.0 \mathrm{~m} / \mathrm{s}$ & $0.47 \mathrm{~m} / \mathrm{s}$ \\
\hline $\begin{array}{l}\text { Battery } \\
\text { capacity }\end{array}$ & $\begin{array}{c}3.7 \mathrm{~V} \\
10.5 \mathrm{Ah}\end{array}$ & - & $\begin{array}{c}3.7 \mathrm{~V} \\
24.2 \mathrm{Ah}\end{array}$ & $\begin{array}{c}3.7 \mathrm{~V} \\
48.0 \mathrm{Ah}\end{array}$ \\
\hline Efficiency & High & Low & Medium & High \\
\hline Runtime & High & Low & High & High \\
\hline $\begin{array}{c}\text { Thrust } \\
\text { generation }\end{array}$ & Low & Low & Medium & High \\
\hline $\begin{array}{c}\text { Diving } \\
\text { capability }\end{array}$ & No & Yes & No & Yes \\
\hline
\end{tabular}

building a robotic fish that is as efficient, agile, and quiet as a natural fish is still far from becoming a reality, the authors in [2] estimate, that the possibility of a robot fish able to swim with real fish in open water will get much more likely in the next ten years.

Due to their potentially very high efficiency, anguilliform swimming robots have been the focus of many roboticists, which have used different methods to achieve the traveling wave pattern. The REEL robots mimicking anguilliform locomotion [8], the lamprey-like robot, which is controlled through a neuromuscular interface [9], a different biorobotic platform also inspired by the lamprey [10], the underwater snake robot Mamba, developed at NTNU, which propels using both aquatic locomotion and conventional propellers [11], or the segmented anguilliform swimming robots AmphiBot I-III [12], [13], [14] are just examples of a wide variety, which is more extensively covered in [2]. In this work, the three robots Lampetra Artefact [15], Amphibious snake-like robot [16] and Envirobot [17] were chosen to represent different solutions with very different approaches.

Lampetra Artefact possesses a highly segmented body and uses an innovative actuator based on rotating permanent magnets in every second segment, and shows an impressive degree of biomimicry in every aspect. While this similarity to the natural example serves its purpose as a platform to investigate neuromuscular control and goal-directed locomotion, it is not capable of generating high thrust or achieving high speeds.
Amphibious snake-like robot's segments are longer and house three DC motors each, which are coupled to achieve three rotary degrees of freedom in each joint. While the wheeled paddles around its modules add a little more lateral surface to the robot, it is not enough for effective thrust generation. Due to the length of its modules, that are significantly longer than Lampetra Artefact's, its anguilliform wave form lacks continuity, which reduces the efficiency of its propulsion. A much stronger negative effect on the efficiency however, is created by the use of three motors per joint. The other notable weakness of this robot is its low speed. It was designed to test many different types of gaits for aquatic locomotion, with the quickest one resulting in a velocity of only $0.07 \mathrm{~m} / \mathrm{s}$.

Envirobot shows a completely modular approach with a single DC motor, two battery cells and a motor controller in every segment, which allows a variable length of the entire robot. Both Envirobot and Amphibious snake-like robot use the motors in a very inefficient manner, as they constantly work at low rpm in an oscillating motion and never reach their most efficient speeds. Tests have shown, that the robot's performance increases significantly with its length, but for this comparison a six-module configuration is assumed. With this length it is comparable to the other robots and most performance data has been obtained in this arrangement. In contrast to the other two robots, it has a roughly rectangular cross section with a flat and significantly larger lateral surface, which allows a higher thrust generation.

The different approaches of the presented robots determine their performance in different aspects. While Envirobot achieves a high speed of $1 \mathrm{~m} / \mathrm{s}$, the other two robots are significantly slower. All three robots have been designed to only propel themselves, which is why their thrust generation is mostly only sufficient to overcome their own drag and not enough to carry additional cargo.

Both Amphibious snake-like robot and Envirobot have a low number of modules relative to their length, leading to a rough wave shape. The resulting kinks and edges in the surface of the robot increase its drag, which is why a higher number of segments, as it is implemented by Lampetra Artefact, is preferable.At the same time, the number of actuators should be kept as low as possible, in order to maximize the power to weight ratio.

The actuators and batteries of all three robots are spread out along the whole length of each robot. While this allows an even weight distribution, it increases the mass of the moving body segments, which in turn leads to higher inertia. The opposite can be observed in most anguilliform fish, which have a tapered body and therefore less mass towards the tail. Additionally, the battery capacity is split up into many small cells, which have a lower energy density compared to larger cells. The control of the robots is managed by central pattern generators (CPGs), a neural network inspired by nature, or serpenoid curves, which are responsible for the shape of the body undulation. These approaches are useful for research of neuromuscular control and allow a relatively simple gait generation. The last interesting performance aspect is the robot's ability to dive. While this feature is irrelevant for the propulsion mechanism itself, swimming underwater is 
expected to be more efficient than at or just below the surface, as a lot of energy is dissipated in the creation of waves on the surface [18], [19]. A diving robot therefore opens up more possibilities to investigate its efficiency at different depths and water pressures. While Amphibious snake-like robot is technically capable of diving thanks to its three-dimensional gaits, it is not designed for efficient movement.

\section{DESIGN}

Based on the identified advantages and drawbacks of the presented robots, which are summarized in table I, a new robot has been developed. In order to find an efficient propulsion technology as a possible alternative to marine propellers, our focus is on movement efficiency, thrust, speed, and controllability. High thrust is essential for a propulsion technology to enable the transport of payload, while a reasonable speed is a requirement for most applications. Because the function of propellers is purely based on their shape, their only control parameters are their rotational speed and in some cases the pitch of their blades. A similarly simple control enables a seamless integration into most applications, which can represent a strong advantage over alternative concepts. For comprehensive testing of the concept's performance, the robot is also required to move using its own power and be capable of steering and diving. Lastly, the robot should ideally be able to switch between different gaits, as tests with previously developed anguilliform robots have shown the strong influence of the shape of the traveling wave on their performance and efficiency.

In order to increase thrust and efficiency, the new design leverages a large lateral surface with a continuous wave shape, reduces the number of actuators, and minimizes the mass of moving parts. To achieve this, the robot features a propulsion system that was adapted from the $S A W$ robot developed by David Zarrouk and colleagues [20]. SAW (Fig. 2) is a small robot that explored the efficient use of a traveling wave for propulsion on land. Its core component is a horizontally oriented 3D-printed helix, that is constantly rotating around its central axis, actuated by a small electric motor. The helix runs in the middle of a chain of links that are designed to cancel out the lateral movement, but follow the vertical motion. Through this setup, the links project the rotation onto the vertical plane, creating a traveling wave. When the links provide enough traction on the ground, the robot moves in the opposite direction of the traveling wave. To control its heading, a set of wheels has been added, that steer the robot using a small servo. A better depiction of the actuation mechanism can be found in the original paper.

The $S A W$ concept demonstrates an interesting kinematic to create a continuous traveling wave using only a single actuator, which optimizes the power to weight ratio and drastically simplifies the control of the robot. Wrapped in a flexible waterproof skin, it is also capable of swimming and generating thrust in the water. The overall design however is not suitable for efficient use in water, which is why this paper describes the development of an entirely new robot, optimizing the propulsion mechanism for anguilliform swimming.

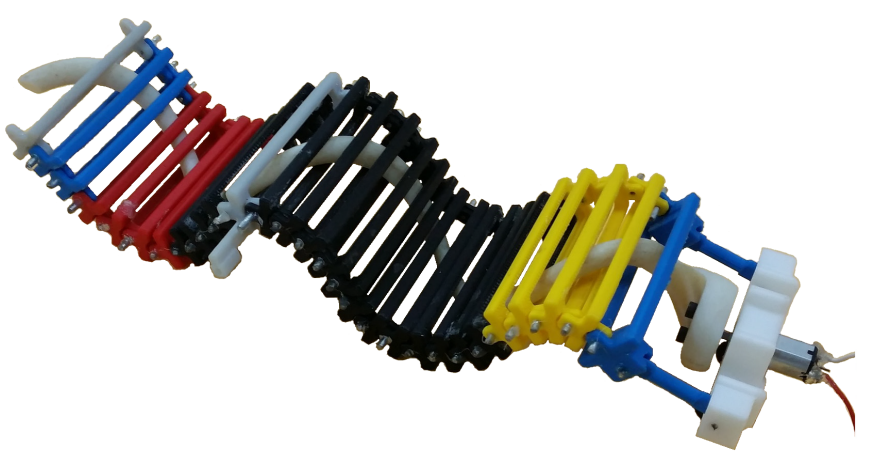

Fig. 2. The Single actuator wave-like robot $(S A W)$, using a rotating helix to create a traveling wave with only a single actuator. The $3 \mathrm{D}$ printed helix is powered by a small electric motor mounted in the front. The coloured links then project the three dimensional movement of the helix onto the vertical plane and provide the traction on the ground to propel the robot forwards. Adapted from [20].

The prototype resulting from this development goes by the name MAR (Marine Anguilliform Robot) and is shown in Fig. 3. It has a total stretched out body length of 108 $\mathrm{cm}$ at a width of only $5.5 \mathrm{~cm}$ and a height of $25 \mathrm{~cm}$. The total weight with the maximum battery capacity of $48 \mathrm{Ah}$ at $3.7 \mathrm{~V}$ amounts to $7500 \mathrm{~g}$. The overall dimensions of the robot are largely dictated by mechanical constraints of the propulsion kinematic. The length was chosen to facilitate one full undulation wavelength at a sufficiently large amplitude, while keeping the maximum angle between two links at a constructively viable level. The height of robot needs to be at least twice the undulation amplitude. Further details about the chosen undulation wave shape follow in section B. To achieve almost neutral buoyancy of the robot and therefore enable it to dive, all individual modules have been designed to be minimally positively buoyant. This was done through careful selection of the used material for each individual part. This approach lead to a well balanced swimming behavior with the upper edge of the robot right at the surface, which can be fine tuned by adding foam or small weights to critical areas.

In the following subsections, the characteristics and design of each module of $M A R$ are presented. Starting with the motor module that drives the helix, the helix that converts rotary motion to lateral undulation, the elements that provide thrust by displacing the surrounding water, the tail which maximizes the output thrust, and finally the head that controls the whole robot and enables steering and diving capabilities.

\section{A. Motor Module}

The motor module (Fig. 4) is located right behind the head, to which it is connected through the joint at its back end. Its primary function is to house the mount for the helix and the propulsion motor unit. Its structure is based on a frame, made from milled POM (Polyoxymethylene) that makes up the bottom, top, front and back walls of the module. The space within this frame is divided into the gearing, the bottom compartment and the top compartment. The gearing is the core of the motor module and is made up of an aluminium 


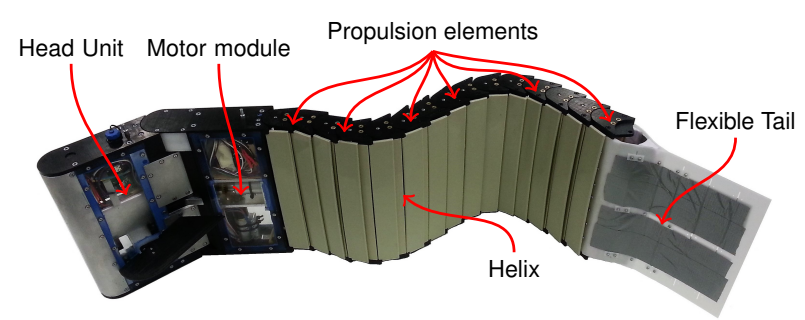

(a) Before adding tail servo motor

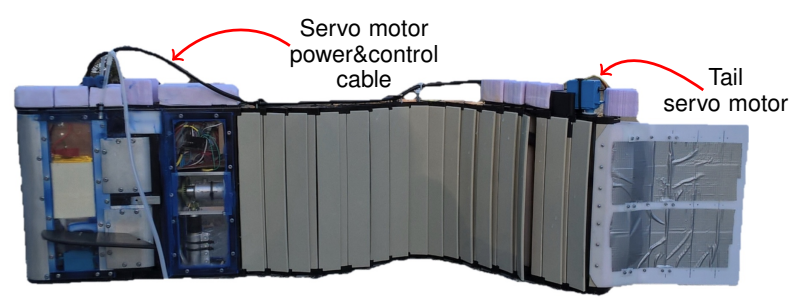

(b) After adding tail servo motor

Fig. 3. An overview of $M A R$ consisting of the head unit, the motor module, the helix hidden by the elements and the flexible tail.

cage, which holds the essential mechanical components. The aluminium helix mount is mounted into the cage using two ball bearings in a fixed and floating bearing arrangement. The helix mount's main function is to transmit the rotation and torque of the motor onto the helix, without putting high stress on individual sections, which would cause it to break. For this reason, it contains a $60 \mathrm{~mm}$ long pipe-like section, into which the helix can be inserted. Due to the close fit around the outer diameter of the helix shaft, forces on the helix get spread over the entire outer surface of the shaft onto the mount. In order to minimize stress concentrations in the helix, the torque is transmitted through an aluminium sheet, that traverses through slots in the mount and the helix, increasing the surface area of the load. The transmission between the helix mount and the motor is realized through brass bevel gears with a transmission ratio of $3: 1$, lowering the rotational speed and increasing the torque. The small input gear is mounted directly to the output shaft of the motor unit, which is bolted to the underside of the gearing cage. Fig. 5 depicts the first test setup of the actuation unit, clearly showing the involved components.

The bottom compartment houses the motor unit which is attached to the underside of the gearing cage. The unit consists of a brushless $90 \mathrm{~W}$ motor by Maxon, a 14:1 gearhead and an encoder. As the motor is used at maximum capacity, temperature regulation is an issue, which is worsened by the placement of two batteries along the sidewalls next to it. To prevent a potentially catastrophic heat build-up, which could damage the motor and the batteries, a heat sink was designed, which conducts the heat of the motor to the underside of the motor module, where a large cooling plate facilitates effective cooling through convection with the surrounding water.

The motor unit and the batteries are connected to two PCBs in the top compartment, which hold the motor controller, an Arduino Pro Mini and the power management of the motor module. They are mounted with enough distance to the sidewall, that two additional battery cells can be added on either side. To enable the communication with the head

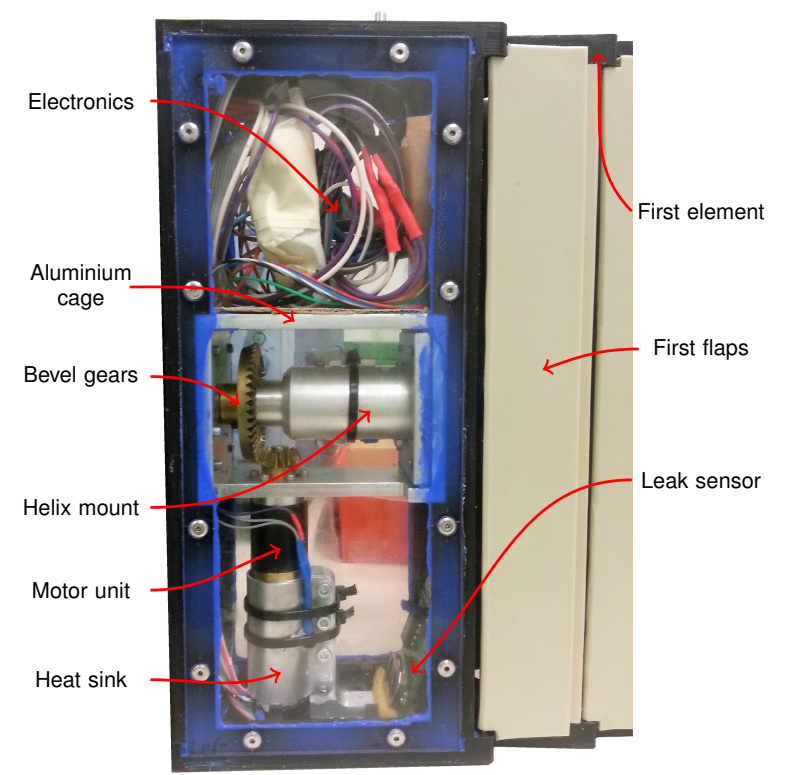

Fig. 4. The motor module without batteries. The top compartment contains electronics including the motor controller and the Arduino. The bevel gears mounted inside the aluminium cage transmit the power to the helix mount, using the motor unit mounted underneath. To cool the motor, a heatsink, which includes a temperature sensor, transmits the excess heat to the surrounding water. Directly behind the motor module the first element is mounted with the first flaps, bridging the gap between module and element. For the first tests, a wireless leak sensor was integrated into the robot.

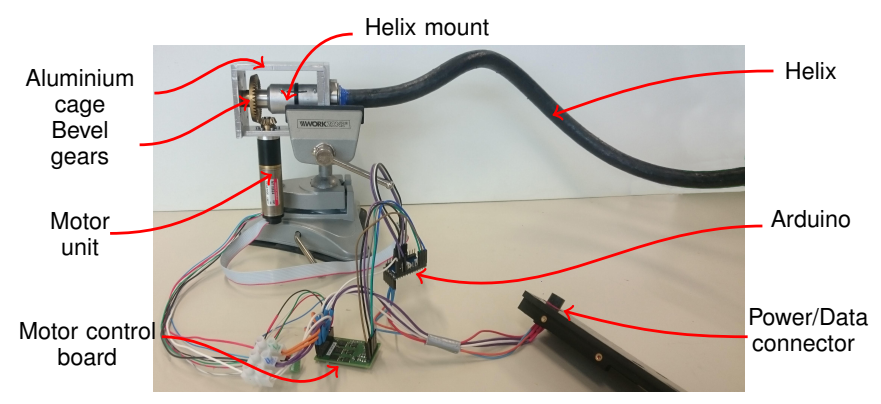

Fig. 5. The first functional model comprises the aluminium profile, which holds the bevel gears and the motor unit with gearhead and encoder. The helix is mounted in the aluminium helix mount. The motor is controlled using the motor control board, which is connected to the Arduino.

module, an IP68 rated connector is mounted at the top of the front wall, where it directly connects to its counterpart on the head module.

The side walls of the motor module are made from $3 \mathrm{~mm}$ thick plexiglass, that allow the monitoring of all processes on the inside. This attribute is particularly important in the motor module to check the correct function and integrity of the mechanical components involved in the gearing without having to open and reseal the robot.

\section{B. Helix}

The helix is the core component of the entire robot and enables the novel approach for efficient swimming. While the general locomotion of this robot is bio-inspired, the shape 


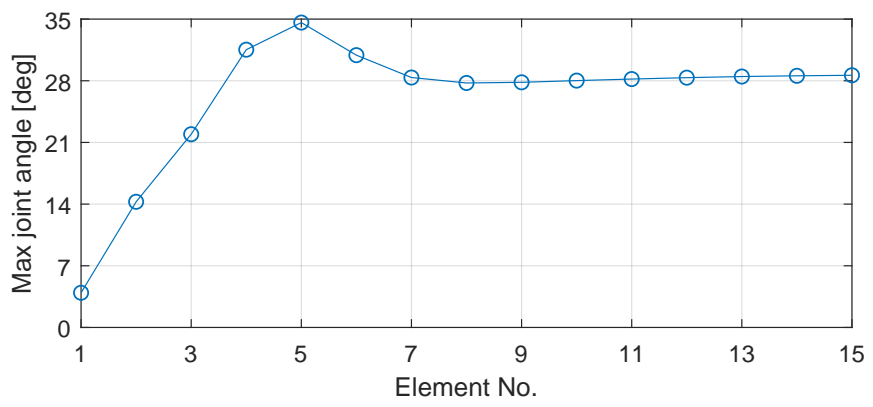

Fig. 6. Maximum joint angles after fixing the parameters $k_{1}(1)$ and $k_{2}(2)$.

of the helix is mainly dictated by mechanical constraints of the overall design and not a direct copy of nature. The key goal was to create a traveling wave, one wavelength long, with an increasing and sufficiently large amplitude to create significant thrust (Fig. 7), as these are typical characteristics for anguilliform swimming. At the same time, the mechanical stress on the helix and the requirements regarding the range of motion of the encasing elements needed to be considered (Fig. 5).

The basis for the helix is a regular helix, based on a sine and a cosine curve in orthogonal planes, with an amplitude of $A=0.09 \mathrm{~m}$ and a wave length of $\lambda=0.5 \mathrm{~m}$. As this helix winds around its central axis in a distance of $A$, its shape has to be modified in a way that its amplitude and therefore its distance from the central axis decreases towards the origin. This shape is also closer to the natural movement, as anguilliform swimming fish show an increasing amplitude of body undulation towards their caudal fin.

To obtain a helix with an increasing amplitude that saturates quickly and starts at zero in the origin, the base helix was multiplied by $k_{1}$ which is a hyperbolic tangent function (1).

$$
k_{1}(x)=A \tanh \left(h_{1}\left(x+\delta_{1}\right)\right)
$$

In this configuration, the helix is technically mountable, but the angle at which it meets the central axis in the origin is very steep. As all the torque exerted on the helix is transmitted through this point, the transition needed to be smoothened, in order to reduce the stress on the material. For this reason, the second scaling factor $k_{2}$ is used such that the first derivative of the helix along $z$ and $y$ directions approaches zero in the origin. As the hyperbolic tangent transitions from -1 to +1 while passing the origin, it was scaled and shifted to obtain the desired transition from 0 to +1 (2).

$$
k_{2}(x)=\frac{1+\tanh \left(h_{2}\left(x-\delta_{2}+\delta_{1}\right)\right)}{2}
$$

In numerous iterative steps the involved factors of this base equation have been optimized to achieve an almost linear increase of the maximum angle between the first five consecutive elements (Fig. 6). Additionally, the two corrective factors $\delta_{1}=0.013, \delta_{2}=0.1$, and tanh scaling factors $h_{1}=5$, $h_{2}=20$, have been added in (1) and (2) to position the helix ideally in respect to its mount in the motor module and the

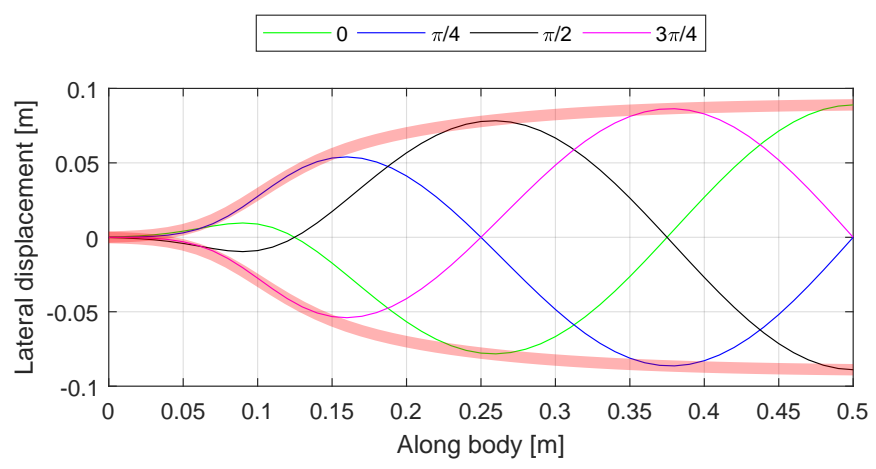

Fig. 7. Lateral displacement of elements along the body imposed by the helix in 4 different phase angles of the motor. The transparent red borders depict the maximum displacement at each point along the body, imposed by $k_{1}$ and $k_{2}$.

first element. This resulted in the following spatial equations of the helix along $y-z$ dimensions as a function $x$, which represents the distance along the body towards the tail.

$$
\begin{aligned}
& y(x)=k_{1}(x) k_{2}(x) \sin (2 \pi x / \lambda) \\
& z(x)=k_{1}(x) k_{2}(x) \cos (2 \pi x / \lambda)
\end{aligned}
$$

The increasing amplitude and the resulting lateral displacement of the helix is depicted in Fig. 7.

The $560 \mathrm{~mm}$ long helix was then manufactured in three parts out of PA2200, a sintering powder based on Polyamide 12 , by selective laser sintering. The connections between the individual parts were centered and reinforced through a tongue and groove design and a Nitinol core and glued together using an epoxy glue. The helix was further encased in two layers of carbon fibre mesh tube of $0.4 \mathrm{~mm}$ and $0.6 \mathrm{~mm}$ thickness and infused with epoxy resin. The last layer consists of a $0.5 \mathrm{~mm}$ thick heat shrink tube with a smooth texture to reduce the friction between the helix and the elements. This extensive reinforcement resulted in a very strong part of 18 $\mathrm{mm}$ thickness, that shows flexible properties under high stress. The manufactured helix is shown as part of the first functional model in Fig. 5.

While the shape of this specific helix certainly leaves room for improvement, it was designed as a first proof of concept to evaluate the drive kinematics. As the helix is easily interchangeable, it can be swapped out for different shapes, that mimic nature more closely in order to maximize the efficiency.

\section{Elements}

The elements are a chain of identical passive components that are actuated by the helix rotating within them. Their setup is shown in Fig. 8.

Each element consists of identical bottom and top holders with high side walls in between and two front-hinged flaps that bridge the gap to the following element. To better integrate the flaps into the element without obstructing the flow of water around it, they are mounted behind the slightly outwards angled side walls. This wedge-like shape allows the flaps 


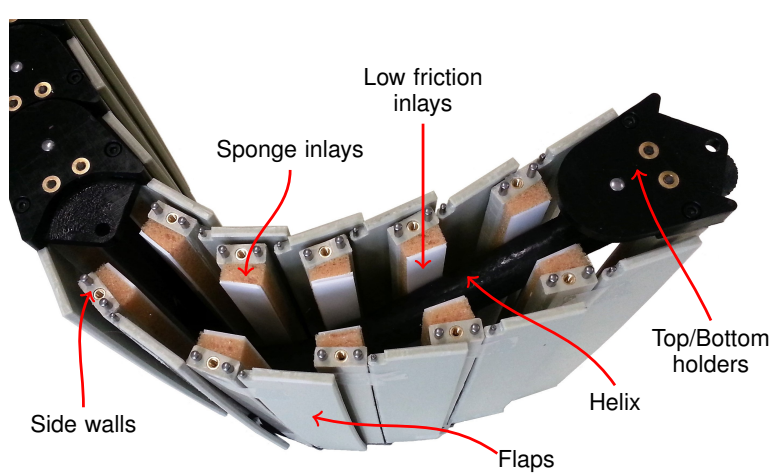

Fig. 8. Structure of the elements of MAR which are comprised of the top and bottom holders, side walls, flaps, low friction inlays and sponge inlays to form parallel contact surfaces for the helix rotating in the middle.

to lie flat on the side wall of the following element at any occurring angle without sticking out to the sides, mimicking the function of fish scales. Because of the rectangular crosssection of the side walls and the angle between them, the contact area between the walls and the helix is reduced to a point contact. While a small contact area reduces the friction, it also leads to reduced stability, as the element can easily pivot around the contact point. This instability is disadvantageous, because it reduces the accuracy with which the elements follow the shape of the helix. This problem is solved through the attachment of low friction inlays made of POM on the inside of the side walls. To amount for the variable space requirements of the helix due to its rotation and compensate the angle of the side walls, the inlays are glued to precisely cut wedge-shaped pieces of sponge. Due to the sponges shape, the inlays are parallel to each other, which allows a line contact between helix and inlay and therefore stabilizes the elements rotation, while its compression allows unrestricted movement with minimized friction.

\section{Tail}

The tail is responsible for most of the propulsion thrust and can be seen in Fig. 9a. It consists of a flexible sheet of plastic that sits at the end of the very last element and acts as the caudal fin.

The most important parameter for this component is its flexibility. If it is too stiff, the fin creates high peaks in the drawn current when it is moving from one side to the other, because it displaces the water to the sides instead of to the rear. If it's too soft, it is simply dragged behind the robot without effectively generating thrust. For good results, the bent tail should roughly follow the curvature of the robot, which requires a decreasing gradient in stiffness from the base of the tail to its tip. This is based on momentum balance calculations, which indicate that matching the resonant frequency of the tail to the undulation frequency increases the force production [21].

To achieve the stiffness gradient and allow an experimental adjustment, the tail flap is made of a $1 \mathrm{~mm}$ thick sheet of POM with multiple vertical slits. These slits increase the flexibility, but can be stiffened by attaching small reinforcement plates
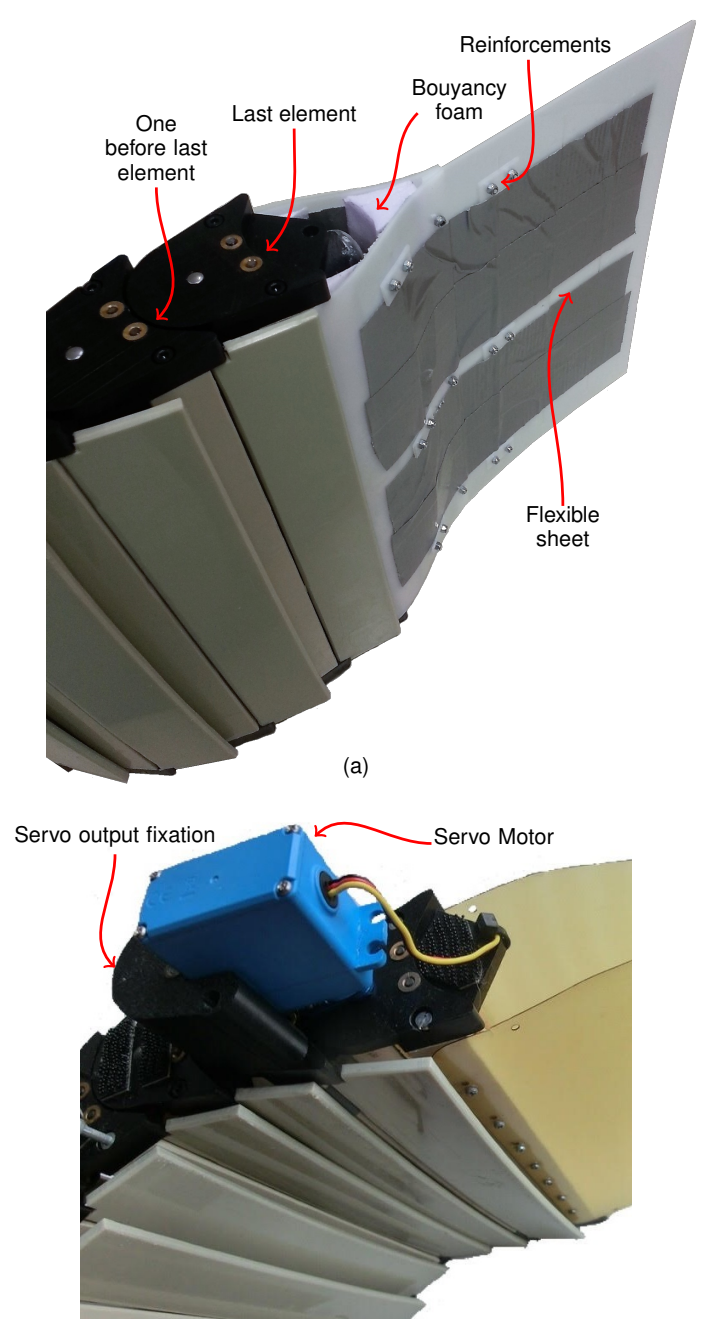

(b)

Fig. 9. (a) The tail of the robot is fastened to the last element and is made from a $1 \mathrm{~mm}$ thick sheet of POM. To achieve neutral buoyancy, a piece of foam is glued to the inside of the stiff part surrounding the tip of the helix. To adjust the stiffness gradient of the flexible tail, small reinforcements are screwed to the flexible sheets. (b) Installed servo motor on the very last element for the actuated tail mechanism.

that bridge them. In preliminary experiments, the number of reinforcements for each slit was determined to obtain an effective stiffness gradient.

After the thrust and speed experiments described in section III, an actuation was added to the tail of the robot, to improve its heading control. The installed servo motor, depicted in figure 9b, allows the addition of an offset on the joint angle between the last two elements. It is fixed on the penultimate element, with its output shaft connected to the axis of the last element. Rotation of the servo motor helps $M A R$ to change its heading by using the tail as a rudder.

\section{E. Head}

The head module houses all components for the extra heading control, in addition to the actuated tail, and communication of the robot (Fig. 10). The back end contains a large vertical joint that connects to the motor module, which allows the 


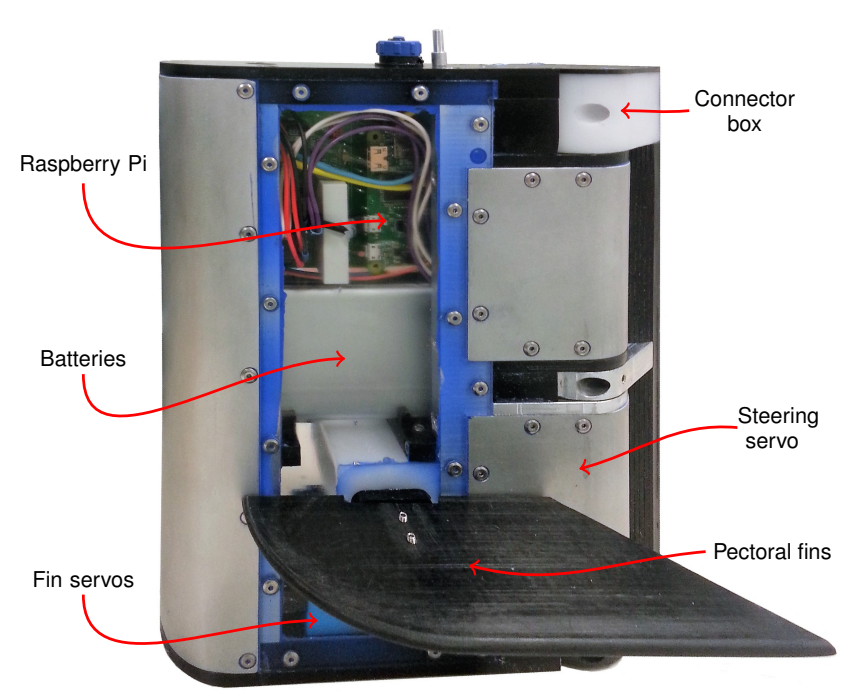

Fig. 10. The head module responsible for the robots autonomy. Clearly visible from the outside are the main board holding the Raspberry Pi (1), the batteries (2), the connector box (4) and the pectoral fins (6). The fin servos (3) are almost completely covered by the fin, while the steering servo (5) is encased in an aluminium wall. On top, the connector for recharging the battery and for an optional air pipe can be seen, while the external WiFi antenna is not mounted yet.

head to turn left and right. This joint is actuated by a servo motor sitting at the bottom of the joint, so that the head can act as a front mounted rudder to control MAR's swimming direction. For communication with the motor module, the top part of the joint contains a connector box, which sits on a hollow axis and features a seven pin connector towards the motor module. Through the axis, cables can be fed to the connector, which allows a free rotation of the joint without any strain on the cables. To enable the robot to dive, two large pectoral fins are attached to either side of the head. These fins are actuated individually by two smaller servo motors sitting side by side in front of the steering servo. Using brass bevel gears, their movement is transmitted onto the fins, allowing pitch and roll movements of the robot through synchronous or opposed rotation. Above the fins, the head module offers space for four $6000 \mathrm{mAh}$ battery cells and two printed circuit boards. The PCBs are mounting platforms for a Raspberry Pi Zero $W$, which functions as the main computing power, as well as the battery charger and all necessary connectors. The Raspberry P $i$ was selected in order to facilitate remote control and troubleshooting via WiFi and serve as a versatile platform for the implementation of autonomy features.

The main structure of the head module is made up of four vertical aluminium beams between the base and top plates, which are made of POM. The outside walls are made from bent aluminium sheets for extra toughness and PMMA side walls that allow an unobstructed view of the robot's inside for easier leak detection and monitoring of the electronics.

\section{Autonomy Features}

This section is dedicated to the addition of low and high level controllers to $M A R$, which enable a path following autonomy feature. The design follows the same architecture

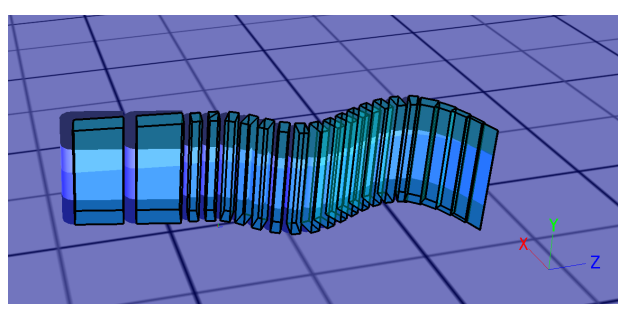

Fig. 11. The simulation model of MAR in Webots.

as depicted in [17], where an inner-loop/outer-loop system is used to decouple the design of the heading and speed controller from the path following controller [22]. These controllers enable a higher level of control of the robot, such as following a desired path. The details of the controller design are out of the scope of this paper, but the results of the path following controller are depicted to demonstrate the viability of autonomy features on the MAR hardware. In the following subsections, a simulation model of MAR is introduced and the simulation results for the swimming speed and the path following capability are shown and compared to experimental data.

\section{A. Simulation Model}

In order to design controllers for MAR and validate different hypotheses, a simulated model of the robot was developed, by adapting the already available model of AmphiBot III [14] and Envirobot [17] in Webots [23]. Although the actuation mechanism of Envirobot and AmphiBot is different from MAR, the oscillatory motion of each element in all three robots is similar. Thus, the helix is modelled by imposing and translating its motion to an oscillatory joint angle motion of each element (Fig. 11.)

\section{B. Path Following}

The inner loop of the path following design consists of the speed and heading controllers. Due to the absence of external disturbances and to minimize the stress on the main undulation motor, an open-loop speed controller is used which keeps the oscillation frequency constant at $0.5 \mathrm{~Hz}$. This achieves an almost constant forward speed of $0.2 \mathrm{~m} / \mathrm{s}$. The heading controller is based on a PI controller that minimizes the absolute error between the actual heading of the robot and the desired heading given by the path following controller. The output of the heading controller actuates the servos in the head and in the tail, thus steering the robot to follow a certain heading. With this it is however not possible to follow an exact path.

This is implemented in a path following controller, which is added on top of the heading controller, in the outer-loop, to let MAR swim along a predefined path. For this, the current position of the robot is determined using the optical tracking system installed above the pool, which is then fed to the controller. The goal of the controller is to minimize the distance between the robot and the planned path and, at the same time, reduce the difference between the current heading 
and the desired heading to zero. More details can be found in [22].

\section{EXPERIMENTS}

To evaluate the performance of $M A R$, a series of experiments has been conducted to determine its efficiency in terms of thrust generation and achieved velocity, as well as an evaluation of the implemented autonomy features. To obtain comparable values regarding the propulsion, the generated forward thrust in a static setup and the free swimming speed of the robot were measured, while simultaneously monitoring its overall power consumption.

The results of the thrust measurements were then compared to the previously presented Envirobot as an anguilliform swimming robot benchmark and the T200 thruster from Blue Robotics [24] as a commercial, low-cost, high-thrust-density thruster. The velocity related results are only compared to Envirobot. The data for comparison to Envirobot has been gathered by [25] in a similar setup which monitored its power consumption during free swimming and in a static thrust measurement.

\section{A. Thrust Measurement}

The amount of thrust that is generated by the robot determines how much cargo it can transport and how easily it can be stopped. If it is high enough, the propulsion mechanism can, for example, be used to propel small vessels that carry payload or cargo. It also ensures that the robot is not instantly immobilized, should it get slightly caught in marine plants or hit light rocks. Thrust experiments were carried out in controlled environment to investigate the maximum thrust, the energy consumption at different thrust levels, and the influence of swimming depth on the results.

The sensor used for the thrust measurements is the resistive $3 \mathrm{~kg}$ aluminium load cell RAPG-003M-A from Loadstar Sensors (Fremont, CA, USA). The power that is consumed by the robot is measured using the $D C 2334 A$ demo board by Linear Technology (Milpitas, CA, USA). It is connected directly at the power source and $\log$ s momentary current and voltage.

To obtain reference values for commercially available thrusters, the $T 200$ thruster by Blue Robotics (Torrance, CA, USA) has been used in previous experiments. The three-bladed thruster is designed for the use on swimming robots and radio-controlled vehicles with a maximum power of $350 \mathrm{~W}$. It operates at up to $16 \mathrm{~V}$, where it generates up to $5.1 \mathrm{~kg}$ of force at a rotational speed of $3800 \mathrm{rpm}$.

To ensure the correct function of the motor, its rotational speed and temperature are monitored using the integrated encoder and an external temperature sensor. The depth is tested in 3 stages, at the surface, submerged halfway between the surface and the bottom and scraping over the bottom of the pool. Because the pool in our laboratory is only $10 \mathrm{~cm}$ deeper than the height of the robot, submerged swimming relates to $5 \mathrm{~cm}$ and bottom swimming to $10 \mathrm{~cm}$ below the surface. Meanwhile, the voltage is kept constant at $15 \mathrm{~V}$, which approximates the average expected voltage level of four $3.7 \mathrm{~V}$

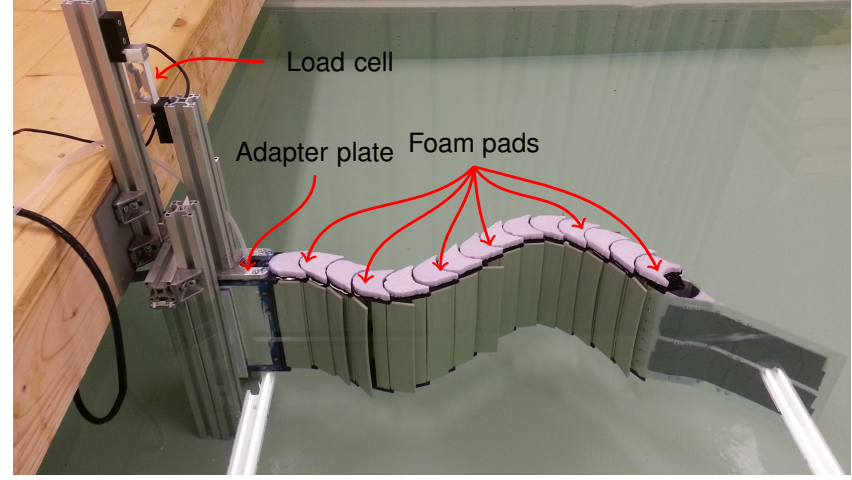

Fig. 12. The static thrust measurement setup built around the load cell to measure the forward thrust of MAR.

batteries in series, as they are used in the robot. The sensor data of the load cell is logged with $500 \mathrm{~Hz}$, while the resolution of the power measurement is limited to $10 \mathrm{~Hz}$.

The analysis of the data follows a simple method. Preliminary tests have shown, that thrust and power consumption are oscillating due to the asymmetry of the helix and the usage of a tail. To acquire meaningful results, each test is therefore run for an extended period of time, so that the oscillation has time to stabilize. Then, a time frame of stable periodic oscillation is selected and the mean value is calculated. This results in a representative average thrust and average power consumption for any given motor speed.

The experiments were carried out in a pool, where the load cell is mounted to a stationary support structure (Fig. 12). Using an aluminium profile and an adapter plate, the motor module is firmly connected to the other end of the load cell. By changing the attachment height of the sensor on the support, the robot can be held in place at different depths. To prevent the robot from exerting undesired forces through turning or rolling, a guidance is placed on both sides, which stabilizes the robot's movement and enables clear force readings in one direction without distortions. For additional stability in the static setup, weights were glued to the bottom of the robot and buoyant foam pads to the top.

In this experiment, the robot was used without the head module or the actuated tail, since no heading control was needed. Instead, a tether was connected to the motor module which transmits all signals and power, to enable precise control over the motor and accurate power measurements outside of the robot. Overall, 27 test runs have been conducted, testing 9 different motor speeds at 3 water levels.

\section{B. Speed Measurement}

The goal for this experiment is to investigate how fast the robot can swim, how much energy it consumes at different speeds and how the swimming depth influences the results. It is expected that swimming below the surface will not increase the speed of the robot, but lower the energy consumption because less energy is lost in the creation of waves on the surface.

Like in the previous experiment, the robot was used in a tethered mode without the head module, in order to monitor the power consumption from the outside. Because this makes 
a heading control and controlled diving impossible, the robot could only swim in a straight line and was weighed down in order to swim below the surface. To measure the velocity and the direction of the freely swimming robot, an optical tracking system is used. It is calibrated to detect a robot-mounted LED at water level, which is powered by a waterproof battery cell stuck to the top of the robot. The power consumption was again measured using the previously presented DC2334A demo board by Linear Technology directly at the power supply.

The optical tracking system used to record the robot's velocity $\log s$ its position at a frequency of $20 \mathrm{~Hz}$, while the power consumption is logged at $10 \mathrm{~Hz}$. While the robot's speed is tested in 9 stages again, the depth is limited to surface and bottom swimming, as the robot was used without the head module containing the pectoral fins. For bottom swimming, the robot was weighed down just enough to make it sink to the floor of the pool, without being subjected to a lot of friction. During both test sets, the supplied voltage is kept constant at $15 \mathrm{~V}$.

In order to calculate the velocity of the robot, its path is logged and plotted. Then, the path where the movement of the robot is unrestricted, is selected for further analysis. As the robot's head constantly swings from side to side during movement, the resulting curve shows a small oscillation. To filter this oscillation and obtain the absolute velocity, a curve is fitted to the plot and its arc length is measured. The average velocity is then calculated based on this distance and the required time. For the determination of the power consumption, a similar process to the first experiment is employed. Like before, the mean value of the power consumption in the free swimming section is calculated to obtain a representative number according to the achieved speed.

The operation of the robot in a tethered mode without the head module enabled a simple test setup, but introduced a major inaccuracy, which is the added drag caused by the flat front face of the motor module and the cable. While the head has a semi-circular front to reduce the drag coefficient, the motor module is completely flat to allow an easy connection to the head. The resulting brick-like shape has a very high drag coefficient, resulting in high losses due to flow resistance. A similar effect is caused by the cable, which connects the robot to the power supply and the control computer. It is approximately $3 \mathrm{~m}$ long and does not float, which significantly adds to the overall drag.

In order to use the available space as effectively as possible, the robot was held in place until the motor had ramped up to the desired speed. Then it was released without any additional impulse and stopped right when it hit a wall. This test was conducted nine times for surface swimming and another nine times for bottom swimming, each time with a different speed.

\section{Model Validation and Path Following}

In order to evaluate the implemented autonomy features, the underlying model is validated by comparing the robot's speed relative to its oscillation frequency in simulation and in free swimming experiments. Then, a path following experiment is conducted to test a real-world application. For this, the robot

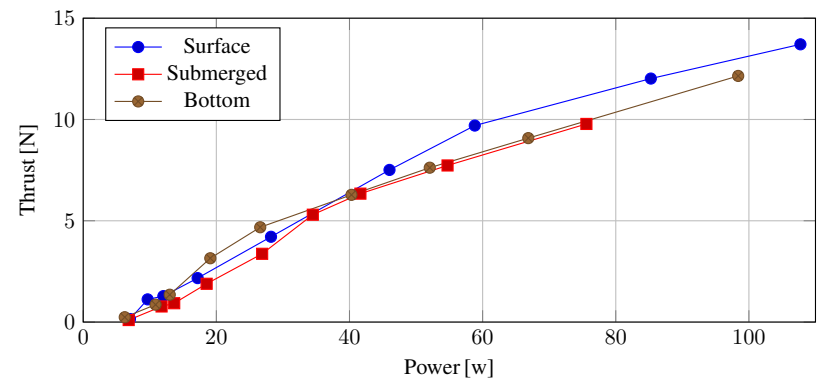

Fig. 13. The generated thrust plotted over the consumed power for all three tested swimming depths. All values are close to each other, but bottom swimming appears to be the strongest below $40 \mathrm{~W}$ and surface swimming generated the most thrust above $40 \mathrm{~W}$.

is tasked to follow an oval shaped path in the lab's pool, while its position is logged by the optical tracking system.

\section{RESUlts}

This section is dedicated to the results of the experiments, analysing the generated thrust, the achieved speed and the robot's efficiency related to thrust and speed. We also present a brief evaluation of the autonomy features in a path following experiment.

\section{A. Thrust Measurement}

Processing of the experimental data of the thrust experiment yielded the mean thrust and power consumption of the robot at different speeds and in different depths. From this, the thrust related efficiency was calculated as the generated thrust over the consumed power. The highest continuously generated thrust of $13.7 \mathrm{~N}$ was achieved swimming at the surface, consuming $107.7 \mathrm{~W}$ of power (Fig. 13). Because this value was determined in a static setup, the robot did not have to overcome its own drag, as it was not moving forwards. This implies that the thrust force usable for applications might be lower than the measured values.

While the mean efficiencies of surface, submerged and bottom swimming show no significant differences, it seems like different swimming depths are preferable for certain speeds. The highest overall measured efficiency at $0.176 \mathrm{~N} / \mathrm{W}$ was achieved when swimming at the bottom with $26.6 \mathrm{~W}$ of power, generating $4.7 \mathrm{~N}$ of thrust. A second peak was reached swimming at the surface with $58.8 \mathrm{~W}$ of power, producing 9.7 $\mathrm{N}$ (Fig. 14) of thrust at an efficiency of 0.165 N/W (Fig. 15). This data implies, that submerged swimming might be more economical at low speeds, while surface swimming presents advantages at higher speeds. However, as the available pool only allowed the robot to be submerged $10 \mathrm{~cm}$ below the surface, wave creation is still a relevant factor, which is why this first experiment is far from conclusive.

Because it yielded the best overall results, the test run at the surface was chosen for the comparison with Envirobot and the T200 thruster (Fig. 14 and Fig. 15). As expected, the new robot generated a much higher maximum thrust than Envirobot and a higher overall efficiency. While the maximum thrust measured with Envirobot was $3.5 \mathrm{~N}, M A R$ reached $13.7 \mathrm{~N}$. The increase 


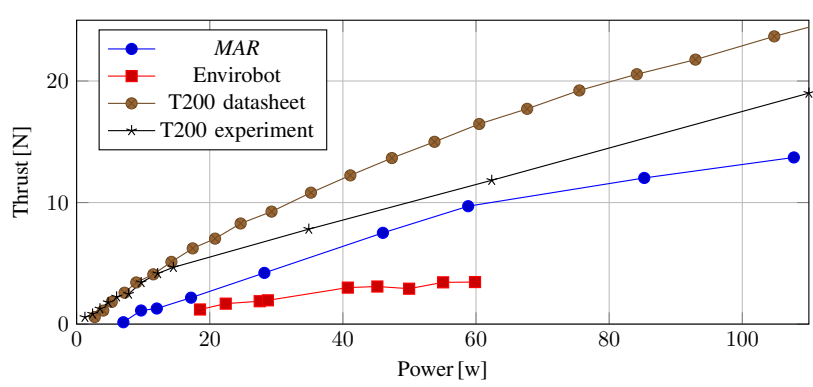

Fig. 14. The generated thrust over the consumed power of MAR, Envirobot and the T200 thruster. For the thruster, this figure shows both the manufacturer's performance figures and the results obtained in the presented experimental setup. While clearly outperforming Envirobot, MAR is not able to compete with the highly optimized thruster yet.

in thrust efficiency ranges from a factor of 1.9 at low speeds to 2.8 at Envirobot's maximum thrust level.

With this performance, MAR came close to the results of the commercial thruster obtained in the same test setup. It appears however, that these results do not match the performance figures published by the manufacturer Blue Robotics, which is possibly due to the age of the used device and an imperfect measurement setup. Although the latter might have also influenced MAR's results, the authors chose to use the manufacturer's performance claims as a benchmark. Bearing the different test conditions in mind, Fig. 14 shows a clear superiority of the highly optimized thruster over the newly developed prototype. It also has to be considered that the $T 200$ is powered by a $350 \mathrm{~W}$ motor operating far below its limits, while $M A R$ is using all its available power stemming from a 90 W motor. Since both the thruster and MAR use brushless DC motors, the motor efficiency is expected to be similar and was not considered for the comparison. Neither were the additional losses due to the gearhead used in the MAR robot.

Fig. 15 also shows a promising aspect of the efficiency curve. While the thruster's efficiency is generally higher, its curve shows a peak at low power and a steady decline with increasing power. MAR's efficiency on the other hand appears to be steadier and reaches its maximum at a much higher power level. With a stronger motor or the right optimizations involving the shape of the helix, friction reduction or the size and stiffness of the tail, it could be possible to raise the entire curve up to the point where MAR beats the thruster regarding thrust efficiency in a certain range of power.

\section{B. Speed Measurement}

The speed experiments have given interesting insight about the robot's maximum speed, the effects of bottom swimming, its performance compared to Envirobot and the impact of drag. Similar to the definition of the thrust efficiency, the speed related efficiency is calculated by dividing the velocity of the robot by its corresponding power consumption.

The maximum speed measured in this experiment was 0.47 $\mathrm{m} / \mathrm{s}$ at $45.5 \mathrm{~W}$, when the robot was swimming at the surface (Fig. 16). The analysis of the power log has shown however, that the power consumption during free swimming is up to 50 $\%$ lower than when the robot is held in place (data not shown).

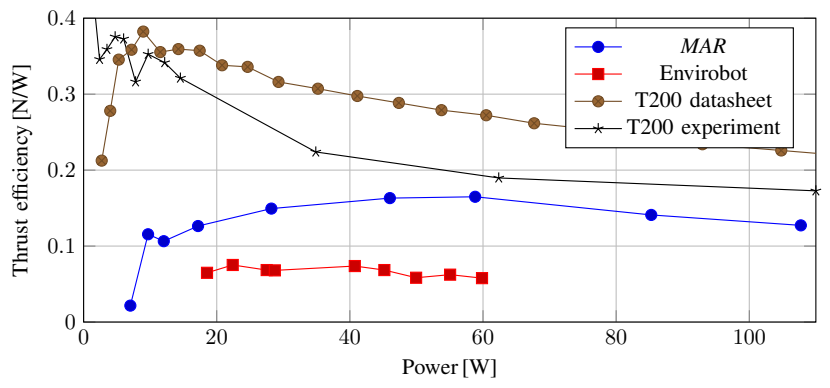

Fig. 15. The thrust efficiency of MAR, Envirobot and the T200 thruster plotted over the consumed power. For the thruster, both the manufacturer's performance figures and the results obtained in the presented experimental setup are shown. While the thruster's efficiency is higher overall, MAR delivers a significantly higher thrust efficiency than Envirobot over the entire power range.

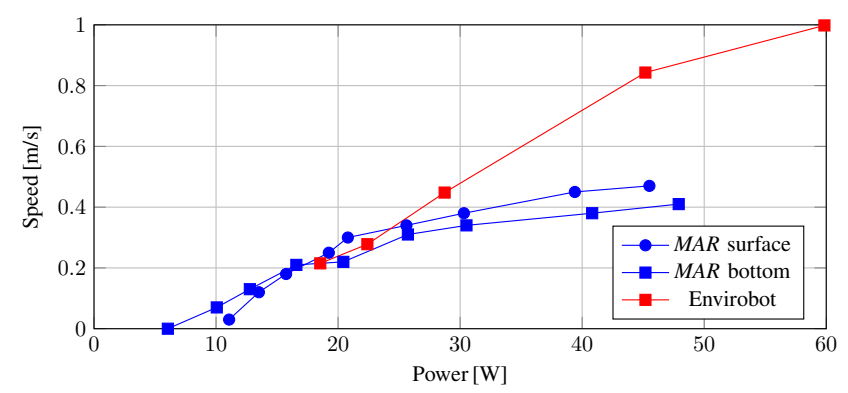

Fig. 16. The swimming speed of $M A R$ versus the consumed power for two cases of surface and bottom swimming.

Due to the spatial limitations of the pool, the robot was kept stationary during the ramp up phase and released after reaching its maximum power. This implies, that the robot could either achieve higher speeds or maintain the measured speeds while carrying a higher payload if the tests were conducted in a larger body of water, where the robot could accelerate to its maximum power while in motion.

The comparison between surface and bottom swimming yielded similar results as the previous experiment. Surface swimming seems to be slightly more efficient overall, but the data shows that bottom swimming is more efficient for very slow speeds. The highest efficiency was reached while swimming at the surface, with $0.0143 \mathrm{~m} / \mathrm{Ws}$ at a power consumption of $20.8 \mathrm{~W}$ (Fig. 17). An explanation for the overall lower efficiency of bottom swimming could be the friction between the robot and the bottom of the pool. In order to reliably keep the robot swimming at the same depth, it was weighed down to make it sit on the floor of the pool. While the weights have smooth convex surfaces at the bottom and their number was carefully adjusted to minimize the overall friction, it might still have played a role in the reduced efficiency of the bottom swimming.

In the comparison of MAR and Envirobot, the latter shows clear benefits regarding its speed by reaching a higher top speed and achieving a higher efficiency for most speeds. At a power consumption of $45.2 \mathrm{~W}$ Envirobot reached a top speed of $0.84 \mathrm{~m} / \mathrm{s}$ versus $M A R$ 's previously mentioned $0.47 \mathrm{~m} / \mathrm{s}$ at $45.5 \mathrm{~W}$. It also achieved a higher maximum efficiency value 


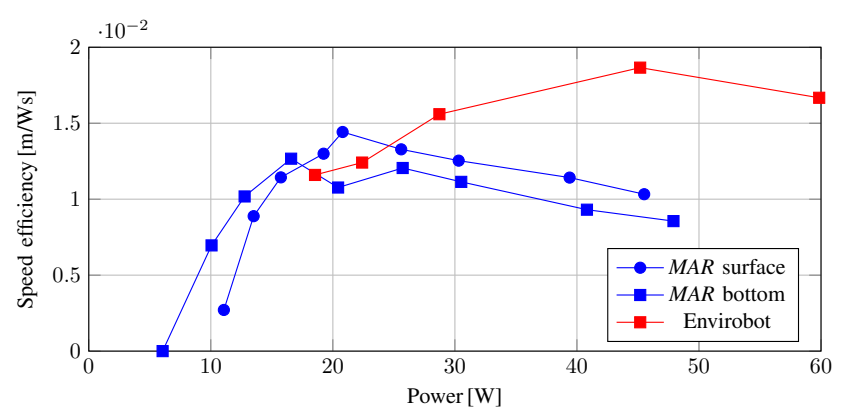

Fig. 17. The swimming speed efficiency of MAR and Envirobot versus the consumed power for two cases of surface and bottom swimming of MAR.

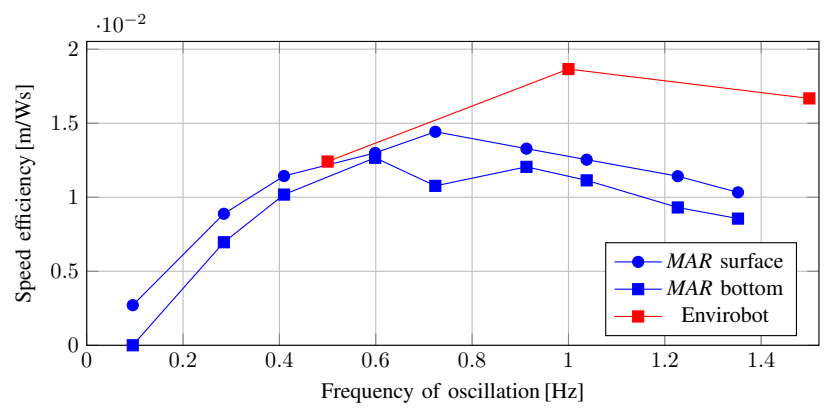

Fig. 18. The swimming speed efficiency of MAR versus the oscillation frequency for two cases of surface and bottom swimming in comparison to Envirobot.

of $0.0187 \mathrm{~m} / \mathrm{Ws}$ over the $0.0143 \mathrm{~m} / \mathrm{Ws}$ of MAR (Fig. 17). While the higher speed is not surprising, as Envirobot shows a much more compact design with minimal drag, the efficiency advantage is unexpected, since $M A R$ was designed to improve on the efficiency using the single motor setup. But in the end both comes down to the high hydrodynamic drag of the new design and its focus on high thrust generation. Another factor to be considered in this comparison is the variable amplitude of Envirobot. As its wave shape is software-controlled, the amplitude could be optimized for maximum speed for each tested level of oscillation. This is a clear advantage over MAR, but also shows the potential gains of using a differently shaped helix for different tasks.

This drag can be quantified by comparing the speed of the traveling wave along the robots body with its overall velocity. As these velocities would be identical under ideal conditions without drag, the difference describes how large the losses are. At its top speed, the robot reached an oscillation frequency of $1.35 \mathrm{~Hz}$, at which the $0.5 \mathrm{~m}$ long helix should propel the robot at a speed of $0.675 \mathrm{~m} / \mathrm{s}$ under ideal conditions. The actual velocity of $0.47 \mathrm{~m} / \mathrm{s}$ indicates a reduction of $30 \%$ due to drag. A great portion of this resistance is caused by the flat front face of the motor module, the drag caused by the $3 \mathrm{~m}$ long tether and the large overall dimensions of the robot. By repeating the test with a mounted head module with a round front face and without a tether, the losses should be reduced significantly. Another possible cause of drag could be the flow of water between and within the elements. As this part of the robot is not waterproof, the whole propulsive section of the robot is filled with water. Because of the overlapping flaps along the

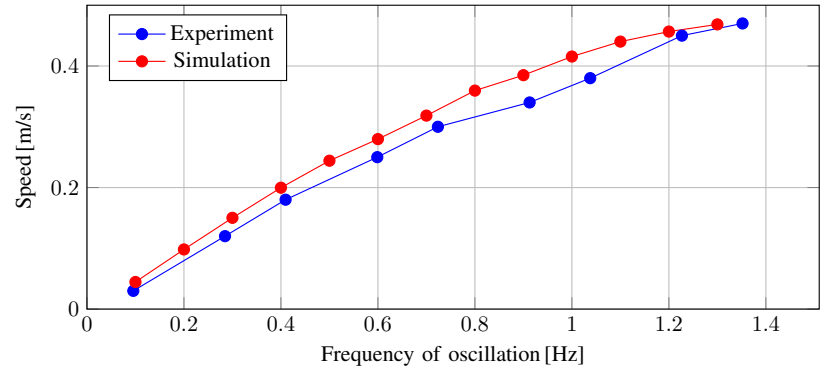

Fig. 19. The comparison of swimming speed of $M A R$ at the surface in simulation versus the experimental results.

outside, which reduce the flow resistance by creating a smooth flat surface, there is no large opening where water could easily flow in during movement. The flaps are however hinged at their front edge, so the water within the robot can easily exit when the robot is in motion. This leads to a vacuum left behind inside the robot, as the outflow is larger than the inflow. This vacuum sucks in the surrounding water through the available openings, which can cause significant drag through turbulence and water currents. While this effect could not be observed or measured, it presents as a valid theoretical explanation that should be investigated in the future.

\section{Model Validation and Path Following}

The results of the open-loop simulations concur with the experiments, as depicted in Fig. 19. It can be observed that the simulation model follows the speed vs. oscillation frequency curve derived from the free swimming experiments in section IV-B.

Fig. 20 depicts the path following results using the simulation model in Webots (Fig. 20a) and in the indoor swimming pool with MAR (Fig. 20b). The desired path was shortened in the experimental setup, to minimize the risk of colliding with the walls of the swimming pool. In both cases, the model and the real robot can follow the path well, but the real robot tends to overshoot in the turns. The simulation model predicts the reality quite well in terms of swimming velocity, but the relationship between the frequency and the turning radius is not as accurate. Adding an internal heading reference unit to replace the direction of movement given by the optical tracking system would also improve the performance of the path following feature.

\section{Vi. CONClusion And Future Work}

While not perfect, the MAR prototype fulfills all set development goals satisfactorily. With 15 elements making up the 0.5 $\mathrm{m}$ long propulsive section, the robot creates a smooth continuous traveling wave for propulsion. This wave is created with a single electric motor by utilising a helix, which drastically increases the power density of the propulsion actuation. Because the motor is continuously running in the same direction at reasonably high rpm, it works efficiently. Since the only control input needed is the motor speed, the control of MAR's propulsion is significantly simplified, compared to generating periodic control signals to many motors as in Envirobot, for 


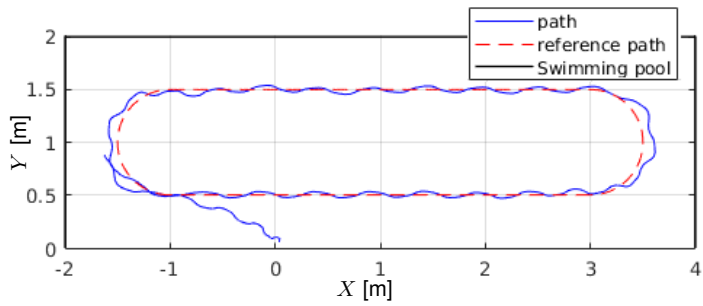

(a) Simulation

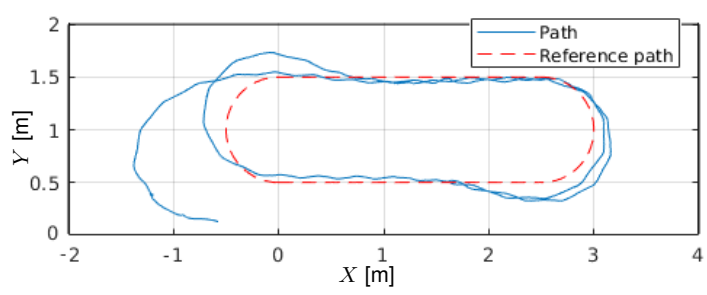

(b) Experiment

Fig. 20. Comparison of the path following function in simulation (a) and the experimental results (b). The length of the oval was reduced for the experiment, to avoid accidental contact with the pool walls.

instance. All batteries are stored inside the head and the motor module, while all moving parts are reduced to structural parts, which reduces the moving mass and therefore the inertia of the system. Since each element performs an oscillating movement, the minimized inertia increases the overall efficiency. Using a turning head, an actuated tail, individually actuated pectoral fins and a well balanced body that is only marginally less dense than water, MAR is capable of steering and diving, as validated by simulation and experimental tests. This allows extended tests in limited spaces and the possibility of investigating the swimming conditions in different depths. To allow optimizations of the robot's gait in the future, the helix is interchangeable. Its mount was designed to minimize the effort to remove the helix and the surrounding hardware of the robot allows helices with greater amplitudes or shortened wavelengths. By adding more of the simply designed elements, the helix and with it the entire robot can also be significantly enlarged. For a further performance increase the length and stiffness of the flexible tail fin will also be systematically optimized. As these two components are the key to the efficiency of the whole locomotion concept, this entails great potential.

The performance tests showed very high thrusts at a moderate power consumption, which promises the possible use of the concept as a propulsor for cargo carrying vessels. The achieved maximum velocity and the speed related efficiency did not fulfill the expectations in the first tests, which can be largely attributed to the spatial limitations and the imperfect test setup. By reducing the robot's drag by running it tetherless with the head module and letting it swim freely in a larger and deeper pool, significantly better results can be expected. There will however always be a trade-off between high speeds and high thrust, determined by the robot's lateral surface and the amplitude of the helix. A smaller surface reduces the drag and more importantly lowers the force exerted on the robot by the surrounding water pressure. Therefore, the same movement can be generated at the same speed with a significantly lower power consumption. The lower force on the other hand implies, that the generated thrust will also decrease. A smaller undulation amplitude has the same effect by shortening the distance that the surrounding water is displaced and decreasing the surface area used to propel the robot forwards. Since $M A R$ was primarily developed to efficiently generate high thrust forces however, the losses regarding speed are acceptable.

To allow a comparison of the overall efficiency of different propulsion concepts, thrust and speed must be taken into account simultaneously. This is possible when using the propulsive efficiency, which is calculated through the multiplication of thrust and speed divided by the consumed power. However, all three properties need to be measured at the same time to obtain generally valid results. This kind of test is planned for the future, to allow an objective comparison of different helix shapes, different robots or commercial thrusters. Further upcoming tests will take place in a larger pool to investigate the true maximum speed and the extent of MAR's diving capabilities. With an update of the onboard electronics a precise power measurement chip could also be integrated into the robot, allowing tetherless real time power measurements.

Another planned experiment to enable a better comparison between propeller propulsion and anguilliform locomotion is the operation of MAR's head modules with a propeller instead of the anguilliform tail. Because the robot was designed to integrate all functional aspects in the front while the back is comprised exclusively of passive components, the entire propulsion mechanism can be exchanged, while still using the same propulsion motor and maintaining all functionality. Therefore, the elements and tail can be dismounted and the helix can be replaced by a shaft holding a propeller, while all other components stay identical.

The next hardware components to be integrated into the robot are an inertial measurement unit (IMU) and a pressure sensor, to give precise information about the current orientation, acceleration and depth. Adding the internal IMU will also help in improving the performance of the heading controller and consequently reduce the overshoot of the path following controller. In combination with the pectoral fins, these sensors can also be used to implement a closed loop stability and depth control.

Since the new robot already showed great potential in its first tests, the further optimization and development will most likely yield even more impressive results. It will reveal new insights on the influence of the swimming depth on the efficiency of fish-like propulsion and use its high efficiency and payload capacity to explore new approaches for autonomous robotic missions.

\section{ACKNOWLEDGMENT}

The authors would like to acknowledge the Swiss National Science Foundation (NCCR Robotics) for the continued support. We would like to thank Thibaut Paschal for sharing experimental results of Envirobot and the thrust measurement setup. 


\section{REFERENCES}

[1] Royal Academy of Engineering, "Future Ship Powering Options: Exploring alternative methods of ship propulsion," Tech. Rep., 2013.

[2] R. Du, Z. Li, K. Youcef-Toumi, and P. Valdivia y Alvarado, Eds., Robot Fish, ser. Springer Tracts in Mechanical Engineering. Berlin, Heidelberg: Springer Berlin Heidelberg, 2015.

[3] M. Sfakiotakis, D. M. Lane, and J. B. C. Davies, "Review of fish swimming modes for aquatic locomotion," IEEE Journal of oceanic engineering, vol. 24, no. 2, pp. 237-252, 1999.

[4] V. van Ginneken, E. Antonissen, U. K. Müller, R. Booms, E. Eding, J. Verreth, and G. van den Thillart, "Eel migration to the sargasso: remarkably high swimming efficiency and low energy costs," Journal of Experimental Biology, vol. 208, no. 7, pp. 1329-1335, 2005.

[5] W. S. Vorus and B. M. Taravella, "Anguilliform fish propulsion of highest hydrodynamic efficiency," Journal of Marine Science and Application, vol. 10, no. 2, p. 163, 2011.

[6] C. Lindsey, "Form, function, and locomotory habits in fish," in Fish Physiology. Elsevier, 1978, pp. 1-100.

[7] D. S. Barrett, "Propulsive efficiency of Robotuna," Ph.D. dissertation, Massachusetts Institute of Technology, 1988.

[8] K. a. McIsaac and J. P. Ostrowski, "Experiments in closed-loop control for an underwater eel-like robot," in Proceedings 2002 IEEE International Conference on Robotics and Automation (Cat. No.02CH37292), vol. 1, no. May. IEEE, 2002, pp. 750-755.

[9] A. Westphal, N. F. Rulkov, J. Ayers, D. Brady, and M. Hunt, "Controlling a lamprey-based robot with an electronic nervous system," Smart Structures and Systems, vol. 8, no. 1, pp. 39-52, Jul 2011.

[10] C. Stefanini, S. Orofino, L. Manfredi, S. Mintchev, S. Marrazza, T. Assaf, L. Capantini, E. Sinibaldi, S. Grillner, P. Wallén, and P. Dario, "A novel autonomous, bioinspired swimming robot developed by neuroscientists and bioengineers," Bioinspiration \& Biomimetics, vol. 7, no. 2, p. 025001, 2012.

[11] P. Liljeback, O. Stavdahl, K. Y. Pettersen, and J. T. Gravdahl, "Mamba - A waterproof snake robot with tactile sensing," in $\{$ IEEE $\} /\{R S J\}$ International Conference on Intelligent Robots and Systems. Institute of Electrical \& Electronics Engineers ( $\{$ IEEE $\}$ ), Sep 2014.

[12] A. Crespi, A. Badertscher, A. Guignard, and A. J. Ijspeert, "AmphiBot I: an amphibious snake-like robot," Robotics and Autonomous Systems, vol. 50, no. 4, pp. 163-175, Mar 2005.

[13] A. Crespi and A. J. Ijspeert, "AmphiBot II: An amphibious snake robot that crawls and swims using a central pattern generator," in International conference on climbing and walking robots (CLAWAR 2006), 2006, pp. 19-27.

[14] M. Porez, F. Boyer, and A. J. Ijspeert, "Improved Lighthill fish swimming model for bio-inspired robots: Modeling, computational aspects and experimental comparisons," The International Journal of Robotics Research, vol. 33, no. 10, pp. 1322-1341, 2014.

[15] S. Yu, S. Ma, B. Li, and Y. Wang, "An amphibious snake-like robot: Design and motion experiments on ground and in water," in 2009 International Conference on Information and Automation. IEEE, Jun 2009, pp. 500-505.

[16] L. Manfredi, T. Assaf, S. Mintchev, S. Marrazza, L. Capantini, S. Orofino, L. Ascari, S. Grillner, P. Wallén, Ö. Ekeberg, C. Stefanini, and P. Dario, "A bioinspired autonomous swimming robot as a tool for studying goal-directed locomotion," Biological Cybernetics, vol. 107, no. 5, pp. 513-527, Oct 2013.

[17] B. Bayat, A. Crespi, and A. Ijspeert, "Envirobot: A Bio-Inspired Environmental Monitoring Platform," in IEEE/OES Conference on Autonomous Underwater Vehicles (AUV), Tokyo, Japan, Nov 2016.

[18] T. M. Williams, "Swimming by sea otters: adaptations for low energetic cost locomotion," Journal of Comparative Physiology A, vol. 164, no. 6, pp. 815-824, 1989.

[19] G. Nicolas and B. Bideau, "A kinematic and dynamic comparison of surface and underwater displacement in high level monofin swimming," Human Movement Science, vol. 28, no. 4, pp. 480-493, Aug 2009.

[20] D. Zarrouk, M. Mann, N. Degani, T. Yehuda, N. Jarbi, and A. Hess, "Single actuator wave-like robot (SAW): design, modeling, and experiments," Bioinspiration \& Biomimetics, vol. 11, no. 4, p. 046004, Jul 2016.

[21] M. C. Leftwich, E. D. Tytell, A. H. Cohen, and A. J. Smits, "Wake structures behind a swimming robotic lamprey with a passively flexible tail," Journal of Experimental Biology, vol. 215, no. 3, pp. 416-425, 2012. [Online]. Available: https://jeb.biologists.org/content/215/3/416

[22] P. Maurya, A. P. Aguiar, and A. Pascoal, "Marine vehicle path following using inner-outer loop control," IFAC Proceedings Volumes, vol. 42, no. 18, pp. 38 - 43, 2009, 8th IFAC Conference on Manoeuvring and Control of Marine Craft.

[23] Webots, "http://www.cyberbotics.com," commercial Mobile Robot Simulation Software. [Online]. Available: http://www.cyberbotics.com

[24] BlueRobotics, "T200 Thruster." [Online]. Available: http://www.bluerobotics.com/store/thrusters/t200-thruster

[25] T. Paschal, "Undulatory swimming vs propeller based," BioRob, EPFL, Tech. Rep., 2017. 